\title{
PULLBACK DE RHAM COHOMOLOGY OF THE FREE PATH FIBRATION ${ }^{1}$
}

BY

\author{
KUO-TSAI CHEN
}

\begin{abstract}
Let $M$ and $N$ be smooth manifolds and let $\bar{B}(A)$ be the reduced bar construction on the de Rham complex $\Lambda(M)$ or a suitable subcomplex $A$ of $M$. For every smooth map $f: N \rightarrow M \times M$, the tensor product $\Lambda(N) \otimes$ $\bar{B}(A)$, equipped with a suitable differential, will yield the correct cohomology for the pullback of the free path fibration $P(M) \rightarrow M \times M$ via the smooth map $f$. Moreover, $\Lambda(N) \otimes \bar{B}(A)$ can be taken as a de Rham subcomplex of the pullback space.
\end{abstract}

Let $M$ and $N$ be $C^{\infty}$ manifolds (or differentiable spaces). Let $P(M)$ be the smooth free path space, i.e. the space of all smooth paths in $M$. The free path fibration of $M$ is the map $\pi_{M}: P(M) \rightarrow M \times M$ sending each path $\gamma: I \rightarrow M$ to the pair $(\gamma(0), \gamma(1))$.

Given a smooth map $f$, consider the pullback diagram:

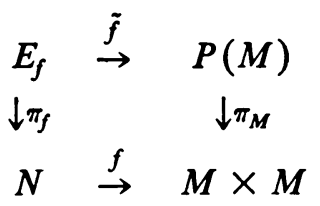

The following examples illustrate the significance of the pullback:

1. If $f: N_{0} \times N_{1} \rightarrow M \times M$ is an inclusion, then $E_{f}$ is the space of smooth paths from $N_{0}$ to $N_{1}$.

2. If $f: M \rightarrow M \times M$ is the diagonal map, then $E_{f}$ is the smooth free loop space of $M$.

3. Let $p: E \rightarrow M$ be a smooth fibration, and let $p^{\prime}: E^{\prime} \rightarrow M^{\prime}$ be the pullback of $p$ via a smooth map $q: M^{\prime} \rightarrow M$. If $f=p \times q$, then $E_{f}$ is of the same smooth homotopy type as $E^{\prime}$. In particular, if $M^{\prime}$ consists of a single

Received by the editors May 31, 1977.

AMS (MOS) subject classifications (1970). Primary 55H20, 58D99; Secondary 53C65, 55F99. Key words and phrases. Differential forms, path spaces, fibration, two sided bar construction, Eilenberg-Moore spectral sequence.

${ }^{1}$ Work supported in part by NSF Grant MCS76-06562. The initial stage of this work was done during a visit at Mathematisches Institut der Universität Bonn, whose hospitality is acknowledged with deep appreciation.

๑) American Mathematical Society 1978 
point, the $E_{f}$ is of the same homotopy type as the fiber of the fibration $p$. Specialized to the case of $f=p \times q$, our main result states that, under suitable conditions, there is an isomorphism

$$
\operatorname{Tor}_{\Lambda(M)}\left(\Lambda(E), \Lambda\left(M^{\prime}\right)\right) \simeq H^{*}\left(E^{\prime} ; R\right)
$$

where $\Lambda$ denotes the de Rham complex functor. Thus this work provides a de Rham realization of a theorem of Eilenberg and Moore [4]. Such an isomorphism was considered earlier by Wu [10] in terms of Sullivan's minimal models and the simplicial category. See also the recent works [5] and [6] of Gugenheim for a more general frame work using the DASH category.

Assume that $M$ is simply connected. Choose a differential graded subalgebra $A$ of the de Rham complex $\Lambda(M)$ such that there is an isomorphism $H(A) \simeq H^{*}(M ; R)$ via integration. Let $\bar{B}(A)$ be the reduced bar construction, whose differential $d_{\bar{B}}$ is given by

$$
\begin{aligned}
d_{\bar{B}}\left[w_{1}|\cdots| w_{r}\right]= & \sum_{1<i<r}(-1)^{i}\left[J w_{1}|\cdots| J w_{i-1}\left|d w_{i}\right| w_{i+1}|\cdots| w_{r}\right] \\
& -\sum_{1<i<r}(-1)^{i}\left[J w_{1}|\cdots| J w_{i-1}\left|J w_{i} \wedge w_{i+1}\right| w_{i+2}|\cdots| w_{r}\right] .
\end{aligned}
$$

Here our sign convention differs somewhat from the usual one as found in [7], and $J w=(-1)^{\operatorname{deg} w} w$ for any homogeneous element $w$.

Denote by $\Lambda(N) \otimes_{f} \bar{B}(A)$ the tensor product $\Lambda(N) \otimes \bar{B}(A)$ equipped with a differential $d_{f}$ such that

$$
\begin{aligned}
d_{f}\left(v \otimes\left[w_{1}|\cdots| w_{r}\right]\right)= & d v \otimes\left[w_{1}|\cdots| w_{r}\right]+J v \otimes d_{\bar{B}}\left[w_{1}|\cdots| w_{r}\right] \\
& -J v \wedge f^{*}\left(w_{1} \times 1\right) \otimes\left[w_{2}|\cdots| w_{r}\right] \\
& +(-1)^{\operatorname{deg} u^{\prime} \operatorname{deg} w_{r}} J v \wedge f^{*}\left(1 \times w_{r}\right) \otimes J u^{\prime},
\end{aligned}
$$

where $u^{\prime}=\left[w_{1}|\cdots| w_{r-1}\right]$. Then $\Lambda(N) \otimes_{f} \bar{B}(A)$ is a differential graded vector space (as a matter of fact, a differential graded algebra). The purpose of this paper is to prove the next theorem.

THEOREM 0.1. Let $M$ and $N$ be $C^{\infty}$ manifolds. If $M$ is simply connected and has finite Betti numbers, then there is an isomorphism

$$
H\left(\Lambda(N) \otimes_{f} \bar{B}(A)\right) \simeq H^{*}\left(E_{f} ; R\right) .
$$

It turns out that $E_{f}$ is a differentiable space and has a de Rham complex $\Lambda\left(E_{f}\right)$. The differential graded vector space $\Lambda(N) \otimes_{f} \bar{B}(A)$ can be embedded in $\Lambda\left(E_{f}\right)$ as a subcomplex and the isomorphism (0.3) will be obtained via integration. Therefore the above theorem is of the de Rham type.

In the extreme case of $N=\left\{y_{0}\right\}$ and $f_{0}=\left(x_{0}, x_{0}\right), E_{f}$ is the smooth loop space $\Omega M$ at $x_{0}$, and the isomorphism (0.3) becomes

$$
H(\bar{B}(A)) \simeq H^{*}(\Omega M ; R),
$$


which is a loop space de Rham theorem. (See [3, Theorems 2.3.1 and 4.1.1] and [5, Proposition 6].) At the other extremity of $M=\left\{x_{0}\right\}$, Theorem 0.1 is simply the usual de Rham theorem.

The starting point of our proof of Theorem 0.1 is the local validity of the theorem on $N$ because of the mentioned loop space de Rham theorem. The global validity on $N$ is then obtained through a method of spectral sequences and fine sheaf resolutions. Theorem 0.1 has been announced in [3]. Our original proof is based on a Weil's method of pairing via integration [10].

REMARK. In the category of topological spaces and under very mild conditions, the cohomology of a Serre fibration can be determined through a construction using minimal models. (See [11, Chapter 20].) In comparison with the above result, Theorem 0.1 is more restrictive but relates directly to analysis through integration.

$\$ 1$ contains some direct applications of Theorem 0.1 to the case of $M=$ $S^{n}$. In $\S 2$, the complex $\Lambda(N) \otimes_{f} \bar{B}(A)$ is realized as a subcomplex of the de Rham complex $\Lambda\left(E_{f}\right)$ in order to make integration possible. The isomorphism $(0.3)$ is obtained by pairing terms of certain spectral sequences on $E_{1}$ and $E_{2}$ levels. In $\S 3$, a spectral sequence for $\Lambda(N) \otimes_{f} \bar{B}(A)$ and a related fine sheaf resolution are discussed briefly. In $\S \S 4$ and 5 , we set up geometrically another fine sheaf resolution. The proof of Theorem 0.1 is completed in $\$ 6$ by comparing the two resolutions.

Let $C_{*}$ denote the normalized smooth cubical chain complex functor (on the category of differentiable spaces). The degeneracy will occur on the last coordinate. Let $H_{*}$ (resp. $H^{*}$ ) denote the smooth homology (resp. cohomology) functor. Let $H_{D R}^{*}=H \Lambda$ denote the de Rham cohomology functor. We shall use [-] to denote a coset, which is often a homology or cohomology class. We shall work with the real number field.

1. Some applications. Equip the tensor product $\Lambda(N) \otimes \bar{B}(A)$ with a fibration $\mathfrak{F}$ such that

$$
\mathfrak{F}^{p}(\Lambda(N) \otimes \bar{B}(A))=\sum_{q>p} \Lambda^{q}(N) \otimes \bar{B}(A) .
$$

Then $\Lambda(N) \otimes_{f} \bar{B}(A)$ becomes a filtered differential graded vector space with an associated spectral sequence $\left\{S_{r}^{\prime}, d_{r}\right\}_{r} \geqslant 0$. We have

$$
S_{1}^{\prime p}=\Lambda^{p}(N) \otimes H(\bar{B}(A))
$$

and, owing to Lemma 3.1 ,

$$
S_{2}^{\prime p}=H_{D R}^{p}(N) \otimes H(\bar{B}(A)) .
$$

It follows from (1.3) that, in computing $H\left(\Lambda(N) \otimes_{f} \bar{B}(A)\right)$, we may replace $\Lambda(N)$ by a subcomplex $L$ satisfying the following conditions:

(a) The inclusion induces an isomorphism $H(L) \simeq H_{D R}^{*}(N)$. 
(b) The complex $L$ is closed under the $A \times A$-action, i.e. $L \wedge f^{*}(A \times A) \subset$ L.

Consider the case of $M=S^{n}, n>1$. Choose $A$ to be the 2-dimensional subalgebra of $\Lambda\left(S^{n}\right)$ generated by 1 and $w \in \Lambda^{n}\left(S^{n}\right)$ such that $\int_{s^{n} w}=1$. Then the reduced bar construction $\bar{B}(A)$ has a trivial differential and a basis $\left\{u_{s}\right\}_{s>0}$ with $u_{0}=[], u_{1}=[w], u_{2}=[w \mid w], \ldots$ and $\operatorname{deg} u_{s}=(n-1) s$. Set $u_{s}=0$ for $s<0$. According to (0.2), we have $d_{f}\left(1 \otimes u_{s}\right)=W \otimes u_{s-1}$ where $W=f^{*}(-w \times 1+1 \times w)$.

If $f:\{0\} \rightarrow S^{n} \times S^{n}$ is the point map given by $f(0)=\left(x_{0}, x_{0}\right)$, then $E_{f}=\Omega S^{n}$. It follows from Example 2.3.1 and Theorem 4.1.1 [3] that there is an isomorphism

$$
H(\bar{B}(A))=\bar{B}(A) \simeq H^{*}\left(\Omega S^{n} ; R\right)
$$

so that $\left\{u_{s}\right\}_{s>0}$ represent a basis for $H^{*}\left(\Omega S^{n} ; R\right)$.

EXAMPLE 1 . If $N_{0}$ and $N_{1}$ are submanifolds of $S^{n}$ of positive codimension and if $f: N_{0} \times N_{1} \rightarrow S^{n} \times S^{n}$ is the inclusion, then $W=0$ so that the real cohomology of the differentiable space of smooth paths from $N_{0}$ to $N_{1}$ in $S^{n}$ is isomorphic to the tensor product

$$
H^{*}\left(N_{0} ; R\right) \otimes H^{*}\left(N_{1} ; R\right) \otimes H^{*}\left(\Omega S^{n} ; R\right) .
$$

EXAMPLE 2. If $f: S^{n} \rightarrow S^{n} \times S^{n}$ is the diagonal map, then $f^{*}(w \times 1)=f^{*}(1$ $X w)=w$ and $W=0$ so that the real free loop space cohomology of $S^{n}$ is isomorphic to the tensor product $H^{*}\left(S^{n} ; R\right) \otimes H^{*}\left(\Omega S^{n} ; R\right)$. (D. Sullivan [8] has provided a general algorithm for the computation of the rational free loop space cohomology. See also [9].)

EXAMPLE 3. If $f: S^{n} \rightarrow S^{n} \times S^{n}$ is given by $x \mapsto(x,-x)$, then $W=-w$ $+(-1)^{n} w$. When $n$ is even, then $W=0$, and we are in the same situation as in the last example. When $n$ is odd, then $W=-2 w$. Instead of $\Lambda\left(S^{n}\right)$ $\otimes_{f} \bar{B}(A)$, consider the compley $\left\{A \otimes \bar{B}(A), d_{f}\right\}$, which has a basis consisting of $1 \times u_{s}, w \times u_{s}, s \geqslant 0$, with $d_{f}\left(1 \otimes u_{s}\right)=-2 w \otimes u_{s-1}$ and $d_{f}\left(w \otimes u_{s}\right)=0$. Thus $H^{*}\left(E_{f} ; R\right)=R$.

EXAMPLE 4. In the general case, there is a short exact sequence of differential preserving graded maps

$$
0 \rightarrow \Lambda(N) \otimes R \rightarrow \Lambda(N) \otimes \bar{B}(A) \rightarrow \Lambda(N) \otimes \bar{B}(A) \rightarrow 0
$$

where the third arrow is given by $v \otimes u_{s} \mapsto v \times u_{s-1}$. The resulting long exact sequence is

$$
\begin{aligned}
\ldots & \rightarrow H^{q}(N ; R) \rightarrow H^{q}\left(E_{f} ; R\right) \\
& \rightarrow H^{q-n+1}\left(E_{f} ; R\right) \rightarrow H^{q+1}(N ; R) \rightarrow \ldots .
\end{aligned}
$$

In particular, if $p: E \rightarrow S^{n}$ is a smooth fibration, then, for

$$
f=p \times q: E \times\{0\} \rightarrow S^{n} \times S^{n},
$$


we may replace, in the above long exact sequence, $N$ by $E$ and $E_{f}$ by the fiber $F$. Thus (1.4) generalizes Wang's exact sequence over $R$.

2. A pairing by integration. We are going to equip $E_{f}$ with a differentiable structure and to realize $\Lambda(N) \otimes_{f} \bar{B}(A)$ as a subcomplex of $\Lambda\left(E_{f}\right)$. Throughout this paper, $U$ will denote a convex set, by which we mean an $n$-dimensional convex subset of $R^{n}$, where $n$ is finite but arbitrary. See [3] for a more detailed account of the material in this section.

A map $\alpha: U \rightarrow P(M)$ is called a plot of $P(M)$ if the associated map $\phi_{\alpha}$ : $I \times U \rightarrow M$ given by $(t, \xi) \mapsto \alpha(\xi)(t)$ is smooth. A $p$-form $u$ on $P(M)$ is defined to be a rule that associates to each plot $\alpha$ of $P(M)$ a $p$-form $u_{\alpha}$ on $U$ such that, if $\theta: U^{\prime} \rightarrow U$ is a $C^{\infty}$ map of convex sets, then $u_{\alpha \theta}=\theta^{*} u_{\alpha}$. (The dimensions of $U$ and $U^{\prime}$ need not be the same.) Thus there is a de Rham complex $\Lambda(P(M))$.

By a plot of a $C^{\infty}$ manifold $N$, we mean a smooth map of the type $U \rightarrow M$. A plot of $E_{f}$ is defined to be a map $\phi: U \rightarrow E_{f}$ such that the maps $\pi_{f} \phi$ and $\tilde{f} \phi$ are plots of $N$ and $P(M)$ respectively. There are de Rham complexes $\Lambda(N)$ and $\Lambda\left(E_{f}\right)$ defined through plots. The former coincides with the usual de Rham complex on $N$. The map $\pi_{f}$ induces a differential graded map $\pi_{f}^{*}$ : $\Lambda(N) \rightarrow \Lambda\left(E_{f}\right)$ such that, for every plot $\phi$ of $E_{f},\left(\pi_{f}^{*} v\right)_{\phi}=v_{\pi, \phi}$. Similarly the map $\tilde{f}$ induces a differential graded map $\tilde{f}^{*}: \Lambda(P(M)) \rightarrow \Lambda\left(E_{f}\right)$. Let

$$
\Lambda(N) \otimes_{f} \bar{B}(A) \rightarrow \Lambda\left(E_{f}\right)
$$

be the differential graded map given by $v \otimes\left[w_{1}|\cdots| w_{r}\right] \mapsto \pi_{f}^{*} v \wedge$ $\tilde{f}^{*} \int w_{1} \cdots w_{r}$, where $\int w_{1} \cdots w_{r}$ is a form on $P(M)$ called an iterated integral. (It follows from [3, Theorem 4.2.1] that the map (2.1) is injective.)

By a (smooth) $n$-cube of $E_{f}$, we mean a plot of the type $\phi: I^{n} \rightarrow E_{f}$. The boundary of $\phi$ is $\partial \phi=\Sigma_{1<i<n}(-1)^{i}\left(\lambda_{i}^{0} \phi-\lambda_{i}^{1} \phi\right)$, where $\lambda_{i}^{e}, \varepsilon=0,1,1<i \leqslant$ $n$, are the face operators.

If $u$ is a $p$-form on $E_{f}$, define the integral $\int_{\phi} u$ such that $\int_{\phi} u=\int_{I^{n}} u_{\phi}$, which is taken to be 0 when $p \neq n$. Let

$$
\Lambda(N) \otimes_{f} \bar{B}(A) \times C_{*}\left(E_{f}\right) \rightarrow R
$$

be the pairing given by $\left\langle v \otimes\left[w_{1}|\cdots| w_{r}\right], \phi\right\rangle=\int_{\phi}\left(\pi_{f}^{*} v \wedge \tilde{f}^{*} \int w_{1} \cdots w_{r}\right)$. Since the map (2.1) is differential graded, the above pairing induces a map

$$
H\left(\Lambda(N) \otimes_{f} \bar{B}(A)\right) \rightarrow H^{*}\left(E_{f} ; R\right) .
$$

The remainder of this work will be devoted to a proof of the fact that, under the hypothesis of Theorem 0.1, the above map becomes an isomorphism.

3. A sheaf resolution. The next lemma can be proved in a routine way.

LEMMA 3.1. For the spectral sequence $\left\{S_{r}^{\prime}, d_{r}\right\}$ associated to $\Lambda(N) \bigotimes_{f} \bar{B}(A)$, 
the differential

$$
d_{1}: S_{1}^{p}=\Lambda^{p}(N) \otimes H(\bar{B}(A)) \rightarrow \Lambda^{p+1}(N) \otimes H(\bar{B}(A))=S_{1}^{\prime p+1}
$$

is given by $v \otimes[z] \rightarrow d v \otimes[z]$.

Corollary. If $N$ is connected, then

$$
S_{2}^{\prime 0}=R \otimes H(\bar{B}(A)) \subset S_{1}^{\prime 0} .
$$

Let $V$ be an open set of $N$. Regard $\pi^{-1} V$ as the pullback space of the restricted map $f \mid V: V \rightarrow M \times M$. Denote by $\left\{S_{r}^{\prime}(V), d_{r}\right\}$ the spectral sequence of the filtered differential graded vector space $\Lambda(V) \otimes_{f \mid V} \bar{B}(A)$. For $p \geqslant 0$, let $\mathcal{S}^{\prime p}$ be the sheaf of graded vector spaces (over $N$ ) such that

$$
\mathcal{S}^{\prime p}(V)=S_{1}^{\prime p}(V) \simeq \Lambda^{p}(V) \otimes H(\bar{B}(A)) .
$$

Define the sheaf $\mathcal{S}^{\prime-1}$ such that $\mathcal{S}^{\prime-1}(V)=S_{2}^{\prime 0}(V)$. Then $\mathcal{S}^{\prime p}, p \geqslant 0$, can be taken as the sheaf of germs of $H(\bar{B}(A))$-valued $p$-forms on $N$, and the exterior differential $d: \mathbb{S}^{p} \rightarrow \mathcal{S}^{p+1}$ is induced by the spectral sequence differential $d_{1}$ according to Lemma 3.1. The sequence of sheaves

$$
0 \rightarrow{\mathfrak{S}^{\prime-1}}^{\rightarrow}{\mathfrak{S}^{\prime}}^{d} \stackrel{d}{\rightarrow} \ldots \stackrel{d}{\rightarrow}{\mathfrak{S}^{\prime p}}^{d} \rightarrow \ldots
$$

is a fine resolution of the sheaf

$$
\mathfrak{S}^{\prime-1} \simeq \Re \otimes H(\bar{B}(A))
$$

where $\Re$ denotes the constant sheaf. We have, for $p \geq 0$,

$$
H^{p}\left(N, \mathcal{S}^{\prime-1}\right) \simeq H^{p}\left(S^{\prime *}(N), d\right)=S_{2}^{\prime p} .
$$

4. A base-point-free Serre spectral sequence. Hereafter we shall write $E=E_{f}$ and $\pi=\pi_{f}$. Let $\sigma$ be a nondegenerate (smooth) $p$-cube of $N$. A $(p+q)$-cube $\tilde{\sigma}$ of $E$ is called a $\pi$-fibered $q$-cube over $\sigma$ if there is a commutative diagram:

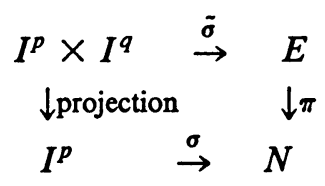

For $1 \leqslant i \leqslant q, \varepsilon=0$, 1, each face $\lambda_{p+i}^{e} \tilde{\sigma}$ is a $\pi$-fibered $(q-1)$-cube over $\sigma$. Set

$$
\partial_{\sigma} \tilde{\sigma}=\sum_{1<i<q}(-1)^{p+i}\left(\lambda_{p+i}^{0} \tilde{\sigma}-\lambda_{p+i}^{1} \tilde{\sigma}\right) .
$$

The totality of $\pi$-fibered cubes over $\sigma$ and the boundary operator $\partial_{\sigma}$ give rise to a normalized chain complex $C(\sigma)_{*}=\left\{C(\sigma)_{q}\right\}$, whose homology will be denoted by $H(\sigma)_{*}=\left\{H(\sigma)_{q}\right\}$. A cycle of $C(\sigma)_{*}$ will be called a $\pi$-fibered cycle over $\sigma$. 
The normalized smooth cubical chain complex $C_{*}(E)$ has an ascending filtration $\mathfrak{F}$ such that $\mathfrak{F}_{p} C_{*}(E)$ has a basis represented by all nondegenerate $\pi$-fibered cubes of $E$ over a nondegenerate cube of $N$ of degree $\leqslant p$. The resulting spectral sequence $\left\{S^{r}, \partial^{r}\right\}_{r} \geqslant 0$ will be called the Serre spectral sequence of the pullback $\pi$. Then

$$
S_{p}^{1}=\amalg H(\sigma)_{*}
$$

summing over all nondegenerate $p$-cubes $\sigma$ of $N$.

If $z$ is a $\pi$-fibered $q$-cycle over $\sigma$, then, for $1 \leqslant i \leqslant p, \varepsilon=0,1, \lambda_{i}^{\varepsilon} z$ is either a $\pi$-fibered $q$-cycle over $\lambda_{i}^{e} \sigma$ or a $\pi$-fibered chain over some nondegenerate cube of degree $<p$. Therefore the differential $\partial^{1}$ is given by

$$
\partial^{1}[z]=\sum(-1)^{\varepsilon+i}\left[\lambda_{i}^{\varepsilon} z\right]
$$

summing over all $(i, \varepsilon)$ with $1 \leqslant i \leqslant q, \varepsilon=0,1$, and $\lambda_{i}^{e} \sigma$ nondegenerate.

Define a $C^{\infty}$ manifold $N$ to be smoothly contractible to $y_{0}$ if there exists a smooth homotopy to $y_{0} \in N$

$$
h: I \times N \rightarrow N
$$

such that $h(0, y)=y$ and $h(1, y)=y_{0}, \forall y \in N$. The next lemma is straightforward.

LEMMA 4.1. If $N$ is smoothly contractible to $y_{0}$, then there exists a commutative diagram

$$
\begin{array}{lll}
I \times E & \stackrel{\tilde{h}}{\rightarrow} & E \\
\downarrow 1 \times \pi & & \downarrow \pi \\
I \times N & \stackrel{h}{\rightarrow} & N
\end{array}
$$

such that $h$ is a smooth contraction as in (4.4) and $\tilde{h}$ is a smooth map with $\tilde{h}(0, \tilde{y})=\tilde{y}, \forall \tilde{y} \in E$.

Regard the trivial map $E_{y_{0}}=\pi^{-1}\left\{y_{0}\right\} \rightarrow\left\{y_{0}\right\}$ as a pullback of the free path fibration so that there is an associated Serre spectral sequence $\left\{S^{r}\left(y_{0}\right), \partial^{r}\right\}$. Then, for $r \geqslant 1$,

$$
S_{0}^{r}\left(y_{0}\right)=H_{*}\left(E_{y_{0}}\right)
$$

and $S_{p}^{r}\left(y_{0}\right)=0$ for $p>0$.

Lemma 4.2. If $N$ is smoothly contractible to $y_{0}$, then the inclusion $C_{*}\left(E_{y_{0}}\right) \subset$ $C_{*}(E)$ induces isomorphisms $S_{p}^{2}\left(y_{0}\right) \simeq S_{p}^{2}, p \geqslant 0$.

Proof. Let $\tilde{\varepsilon}: E \rightarrow E_{y_{0}}$ be given by $\tilde{\varepsilon}(y)=\tilde{h}(1, \tilde{y})$. The composite map $E_{y_{0}} \stackrel{ᄃ}{\rightarrow} E \rightarrow E_{y_{0}}$ induces an identity composite map

$$
S_{p}^{2}\left(y_{0}\right) \rightarrow S_{p}^{2} \rightarrow S_{p}^{2}\left(y_{0}\right), \quad p \geqslant 0 .
$$


On the other hand, the composite map $\tilde{h_{1}}: E \stackrel{\tilde{\varepsilon}}{\rightarrow} E_{y_{0}} \stackrel{\ulcorner}{\rightarrow} E$ induces

$$
S_{p}^{1} \rightarrow S_{p}^{1}\left(y_{0}\right) \rightarrow S_{p}^{1}, \quad p \geqslant 0,
$$

given by $[z] \mapsto\left[\tilde{h}_{1 *} z\right.$, where $z$ is a $\pi$-fibered $q$-cycle over a nondegenerate $p$-cube $\sigma$ of $N$, and $[z] \in H(\sigma)_{q} \subset S_{p}^{1}$. Observe that $h_{1 *} z$ is a linear combination of cubes of the type $\tilde{\sigma} h_{1}$, which is a $\pi$-fibered $(p+q)$-cube over the 0 -cube at $y_{0}$. Thus $\left[h_{1 *} z\right]=0$ for $p>0$.

Let $D \sigma$ be the composite map

$$
I \times I^{p} \stackrel{1 \times \sigma}{\rightarrow} I \times N \stackrel{h}{\rightarrow} N .
$$

If $\tilde{\sigma}$ is a $\pi$-fibered $q$-cube over $\sigma$, define $\tilde{D} \tilde{\sigma}$ to be the composite map

$$
I \times\left(I^{p} \times I^{q}\right) \stackrel{1 \times \tilde{\sigma}}{\rightarrow} I \times E \stackrel{\tilde{h}}{\rightarrow} E,
$$

which is a $\pi$-fibered $q$-cube over the $(p+1)$-cube $D \sigma$. Then $\tilde{D}$ induces maps $H(\sigma)_{q} \rightarrow H(D \sigma)_{q}$, which give rise to maps

$$
\tilde{D}^{1}: S_{p}^{1} \rightarrow S_{p+1}^{1}, \quad p \geqslant 0,
$$

such that

$$
\left(\partial^{1} \tilde{D}^{1}+\tilde{D}^{1} \partial^{1}\right)[z]=\left[h_{1 *} z\right]-[z] .
$$

Hence the lemma is proved.

COROLLARY. Under the same hypothesis as the lemma, there are a long exact sequence

$$
\ldots \rightarrow S_{p}^{1} \rightarrow \cdots \rightarrow S_{0}^{1} \rightarrow S_{0}^{2} \rightarrow 0
$$

and an isomorphism

$$
S_{0}^{2}\left(y_{0}\right) \simeq S_{0}^{2}
$$

induced by the inclusion $E_{y_{0}} \subset E$.

5. The singular cohomological sheaf resolution. For an open set $V$ of $N$, the normalized smooth cubical chain complex $C_{*}\left(\pi^{-1} V\right)$ is filtered with an associated Serre spectral sequence $\left\{S^{r}(V), \partial^{r}\right\}_{r>0}$ if $\pi^{-1} V$ is taken as the pullback space of the restricted map $f \mid V$. Morever,

$$
S_{p q}^{1}(V)=\coprod H(\sigma)_{q}
$$

summing over all nondegenerate $p$-cubes $\sigma$ of $V$.

For $p \geqslant 0$, let $\mathfrak{S}^{p}$ be the presheaf of graded vector spaces over $N$ such that $\mathfrak{S}^{p}(V)=\left\{\mathfrak{S}^{p}(V)^{q}\right\}$ with $\mathfrak{S}^{p}(V)^{q}=\operatorname{Hom}_{Z}\left(S_{p q}^{1}(V), R\right)$. The spectral sequence differential $\partial^{1}$ induces presheaf maps

$$
\delta: \mathfrak{\varsigma}^{p} \rightarrow \mathfrak{S}^{p+1}, \quad p \geqslant 0 .
$$

Denote by $\varsigma^{-1}$ the presheaf such that $\varsigma^{-1}(V)^{q}=\operatorname{Hom}_{Z}\left(S_{0 q}^{2}(V), R\right)$. Since 
$S_{0 q}^{2}(V)$ is a quotient of $S_{0 q}^{1}(V)$, there is a monomorphism

$$
\mathfrak{S}^{-1} \rightarrow \mathbb{S}^{0} \text {. }
$$

Each presheaf $\mathfrak{S}^{p}, p \geqslant 0$, is fine. In fact, let $\left\{U_{i}\right\}_{i \in \mathfrak{B}}$ be a locally finite open covering of $N$ and let $\mu$ be a choice function which assigns to each nondegenerate cube $\sigma$ of $N$ an element $\mu(\sigma)$ of the index set $\mathfrak{P}$ such that $\sigma(0) \in U_{\mu(\sigma)}$. Given $i \in \mathfrak{P}$, define an endomorphism $e_{i}$ of $\mathcal{S}^{p}$ such that, for every open set $V$ of $N, e_{i}(V)$ is the projection

$$
\mathfrak{S}^{p}(V)=\prod \operatorname{Hom}_{z}\left(H(\sigma)_{*}, R\right) \rightarrow \prod^{\prime} \operatorname{Hom}_{z}(H(\sigma), R) \subset \mathfrak{S}^{p}(V),
$$

where the second product $\Pi^{\prime}$ runs over those nondegenerate $p$-cubes $\sigma$ of $V$ with $\mu(\sigma)=i$. Then $\Sigma e_{i}$ is the identity presheaf map of $\mathcal{S}^{p}$, and $\mathcal{S}^{p}$ is fine for $p>0$. Let $\hat{\Im}^{p}$ denote the completion of $\Im^{p}, p>-1$. For $p>0$, each $\hat{\Im}^{p}$ is a fine sheaf.

The presheaf maps (5.1) and (5.2) give rise to a sequence

$$
0 \rightarrow \mathfrak{S}^{-1} \rightarrow \mathfrak{S}^{0} \stackrel{\delta}{\rightarrow} \ldots \stackrel{\delta}{\rightarrow} \varsigma^{p} \stackrel{\delta}{\rightarrow} \ldots
$$

Since $N$ is a manifold, every point $y_{0}$ of $N$ has an arbitrarily small neighborhood $V$ smoothly contractible to $y_{0}$. It follows from (4.6) that the above sequence of presheaves completes to a sequence of sheaves

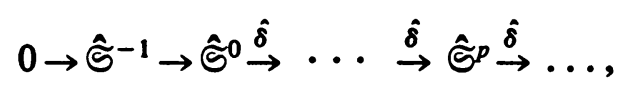

which is a fine resolution of the sheaf $\hat{\Phi}^{-1}$.

Since $N$ is paracompact, $H^{p}\left(\varsigma^{*}(N), \delta\right) \simeq H^{p}\left(\hat{\Im}^{*}(N), \hat{\delta}\right), p>0$. Recall that $\mathfrak{S}^{p}(N)=\operatorname{Hom}_{Z}\left(S_{p}^{2}, R\right)$. The fine resolution (5.4) yields

$$
H^{p}\left(N, \hat{\subseteq}^{-1}\right) \simeq \operatorname{Hom}_{z}\left(S_{p}^{2}, R\right) .
$$

\section{The conclusion of the proof.}

LEMMA 6.1. The pairing (2.2) respects the filtration.

Proof. Let $v$ be a $p^{\prime}$-form on $N$, and let $\tilde{\sigma}$ be a $\pi$-fibered $q$-cube over a $p$-cube $\sigma$ of $N$ with $p<p^{\prime}$. The $p^{\prime}$-form $\sigma^{*} v$ on $I^{p}$ must vanish. The commutative diagram (4.1) implies that $\tilde{\sigma}^{*} \pi^{*} v=0$. Hence

$$
\left\langle\pi^{*} v \wedge \tilde{f}^{*} \int w_{1} \cdots w_{r}, \tilde{\sigma}\right\rangle=\int_{I^{p} \times I^{q}} \tilde{\sigma}^{*}\left(\pi^{*} v \wedge \tilde{f}^{*} \int w_{1} \cdots w_{r}\right)=0 .
$$

In abbreviation, write $\mathfrak{F}^{p}=\mathfrak{F}^{p}(\Lambda(N) \otimes \bar{B}(A))$ and $\mathfrak{F}_{p}=\mathfrak{F}_{p} C_{*}(E)$. Recall that if $\mathfrak{3}^{p}=\left\{u \in \mathfrak{F}^{p}: d_{f} u \in \mathfrak{F}^{p+1}\right\}$, then $S_{1}^{\prime p}=\mathfrak{Z}^{p} /\left(d_{f} \mathfrak{F}^{p}+\mathfrak{F}^{p+1}\right)$ and

$$
S_{2}^{\prime 0}=\left\{u \in \mathfrak{F}^{0}: d_{f} u \in \mathfrak{F}^{2}\right\} /\left(d_{f} \mathfrak{F}^{0}+\mathfrak{Z}^{1}\right) .
$$

Similarly, if $3_{p}=\left\{c \in \mathfrak{F}_{p} ; c \in \mathfrak{F}_{p-1}\right\}$, then $S_{p}^{1}=3_{p} /\left(\partial \mathfrak{F}_{p}+\mathfrak{F}_{p-1}\right)$ and $S_{0}^{2}=3_{0} / \partial 3_{1}$.

The pairing (2.2) induces pairings 


$$
S_{1}^{\prime p} \times S_{0}^{2} \rightarrow R, \quad p \geqslant 0,
$$

and

$$
S_{2}^{\prime 0} \times S_{0}^{2} \rightarrow R
$$

The pairing (6.1) is given by $\langle[u]$, [c] $\rangle=\int_{c} \chi u$, where $u \in 3^{p}, c \in 3_{p}$ and $\chi$ is the map (2.1). Moreover, if $p>0$ and $u^{\prime} \in 3^{p-1}$, then

$$
\left\langle d^{1}\left[u^{\prime}\right],[c]\right\rangle=\left\langle\left[u^{\prime}\right], \partial^{1}[c]\right\rangle \text {. }
$$

If $V$ is an open set of $N$, then, corresponding to (6.1) and (6.2), there are pairings

$$
S_{1}^{\prime p}(V) \times S_{p}^{1}(V) \rightarrow R, \quad p \geqslant 0,
$$

and

$$
S_{2}^{\prime 0}(V) \times S_{0}^{2}(V) \rightarrow R,
$$

which induce presheaf maps

$$
\mathfrak{S}^{\prime p} \rightarrow \mathfrak{S}^{p}, \quad p \geqslant 0,
$$

and

$$
\mathfrak{S}^{\prime-1} \rightarrow \mathfrak{S}^{-1} \text {. }
$$

LEMMA 6.2. If $V$ is smooth contractible to $y_{0} \in V$, then the pairing (6.2a) induces an isomorphism $\mathbb{S}^{-1}(V) \simeq \mathfrak{S}^{-1}(V)$.

Proof. Let $f y_{0}=\left(x^{\prime}, x^{\prime \prime}\right)$. We identify $E_{y_{0}}$ with the differentiable space $P\left(M ; x^{\prime}, x^{\prime \prime}\right)$ of all smooth paths from $x^{\prime}$ to $x^{\prime \prime}$ in $M$. According to the isomorphisms (3.1), (4.5) and (4.7), the pairing (6.2a) can be taken as the pairing

$$
H(\bar{B}(A)) \times H_{*}\left(P\left(M ; x^{\prime}, x^{\prime \prime}\right)\right) \rightarrow R,
$$

which is induced by the pairing

$$
\bar{B}(A) \times C_{*}\left(P\left(M ; x^{\prime}, x^{\prime \prime}\right)\right) \rightarrow R
$$

given by $\left\langle\left[w_{1}|\cdots| w_{r}\right], c\right\rangle=\int_{c} \int w_{1} \cdots w_{r}$.

Let $\gamma$ be a smooth path from $x^{\prime}$ to $x^{\prime \prime}$. Consider the map $g: I \rightarrow M \times M$ given by $t \mapsto\left(x^{\prime}, \gamma(t)\right)$. Let $\pi_{g}: E_{g} \rightarrow I$ be the associated pullback from the path fibration $\pi_{M}$. Then $C_{*}\left(E_{g}\right)$ has an associated Serre spectral sequence $\left\{T^{r}, \partial^{r}\right\}$. Corresponding to the pairing (2.2), there is a pairing

$$
\Lambda(I) \otimes_{g} \bar{B}(A) \times C_{*}\left(E_{g}\right) \rightarrow R
$$

In the place of the pairing (6.2), we now have the pairing

$$
H(\bar{B}(A)) \times T_{0}^{2} \rightarrow R .
$$

The respective fibers of the pullback $\pi_{g}$ above the points 0 and 1 are 
precisely $P\left(M ; x^{\prime}, x^{\prime}\right)$ and $P\left(M ; x^{\prime}, x^{\prime \prime}\right)$. According to (4.7), there are isomorphisms $H_{*}\left(P\left(M ; x^{\prime}, x^{\prime}\right)\right) \simeq T_{0}^{2} \simeq H_{*}\left(P\left(M ; x^{\prime}, x^{\prime \prime}\right)\right)$. This implies that the pairing (6.6) can be, in turn, taken as the pairing

$$
H(\bar{B}(A)) \times H_{*}\left(P\left(M ; x^{\prime}, x^{\prime}\right)\right) \rightarrow R
$$

which is induced by a pairing similar to (6.7). Using Theorem 2.3.1 of [3] (see also [5, Proposition 6]), we obtain an isomorphism

$$
H(\bar{B}(A)) \simeq H^{*}\left(P\left(M ; x^{\prime}, x^{\prime}\right) ; R\right)
$$

Hence the lemma is proved.

Proof of THEOREM 0.1. There is a commutative diagram of sheaves

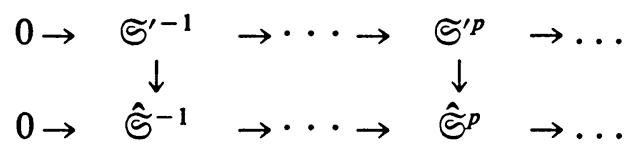

where the first row is the fine sheaf resolution (3.2), the second row is the fine sheaf resolution (5.4), and the vertical maps are induced by (6.4) and (6.5). According to Lemma 6.2, the first vertical map is a sheaf isomorphism so that $H^{p}\left(N, \Im^{\prime-1}\right) \simeq H^{p}\left(N, \hat{\Im}^{-1}\right), p \geqslant 0$. From (3.4) and (5.5), we obtain $S_{2}^{\prime p} \simeq$ $\operatorname{Hom}_{Z}\left(S_{p}^{2}, R\right)$. Thus the pairing (2.2) induces a spectral sequence pairing

$$
S_{r}^{\prime} \times S^{r} \rightarrow R,
$$

which is nondegenerate for $r \geqslant 2$. Since both spectral sequences $\left\{S_{r}^{\prime}, d_{r}\right\}$ and $\left\{S^{r}, \partial^{r}\right\}$ converge, the pairing (2.2) induces a nondegenerate pairing

$$
H\left(\Lambda(N) \otimes_{f} \bar{B}(A)\right) \times H_{*}\left(E_{f}\right) \rightarrow R
$$

Hence the theorem is proved.

\section{BIBLIOGRAPHY}

1. K. T. Chen, Iterated integrals of differential forms and loop space homology, Ann. of Math. 97 (1973), 217-246.

2. __ Reduced bar construction on de Rham complexes, Algebra, Topology and Category Theory, A Collection of Papers in Honor of Samuel Eilenberg, Academic Press, London, 1976, pp. 19-32.

3.

4. S. Eilenberg and J. C. Moore, Homology and fibrations. I, Comment. Math. Helv. 40 (1966), 199-236.

5. V. K. A. M. Gugenheim, On the multiplying structure of the de Rham theory, J. Differential Geometry 11 (1976), 309-314.

6. , On the multiplicative structure of the de Rham cohomology of induced fibrations (preprint).

7. L. Smith, Homological algebra and the Eilenberg-Moore spectral sequence, Trans. Amer. Math. Soc. 129 (1967), 58-93. 
8. D. Sullivan, Differential forms and topology of manifolds, Conferences on Manifolds, Tokyo, 1973.

9. Infinitesimal computations in topology (preprint).

10. W. T. Wu, Theory of $I^{*}$-functor in algebraic topology, Sci. Sinica 18 (1975), 464-482.

11. S. Halperin, Lectures on minimal models, Université des Sciences et Techniques de Lille. I, 1977.

Department of Mathematics, University OF IllinoIs, Urbana, Illinois 61801 